Poster Presentation Abstracts

In the high education stratum this difference was 5 compared to 14 in the low stratum. The same pattern was found for verbal, performal IQ, processing speed and SDQ scores. Neurological outcome of preterm born children was not normal in $49 \%$ especially due to minor neurological dysfunction (MND), which occurred in 44\%. MND nor M ABC scores were influenced by parental education, with comparable preterm-term diferences in each parental education stratum.

Conclusions: The biological risk of preterm children is highlighted by their neurological outcome. The social risk factor modifies this biological risk and determines especially cognitive and behavioral outcome.

\section{8 \\ OBSTRUCTIVE PULMONARY DISEASE IN OLD AGE AMONG INDIVIDUALS BORN PRETERM}

\section{E. Berggren Broström}

\section{Karolinska University Hospital Neonatolgy, Karolinska Insitutet, Stockholm, Sweden}

Background: There are only few studies of the association between preterm birth and risk of chronic lung disease in old age. The aim of this study was to assess the association between poor fetal growth, preterm birth, sex and risk of asthma and Chronic Obstructive Pulmonary Disorder (COPD) in adulthood.

Method: We have followed up a cohort of all infants born preterm ( $<35$ weeks) or with low birth weight (< 2,000 grams and $<2,100$ grams for girls and boys, respectively) in a source population of 250,000 individuals born from 1925 through 1949 in Sweden and an equal number of controls. Follow-up started in January 1st, 1987 and continued to December 31,2006 . At start of follow up, there were 6,425 subjects in the cohort. There were 2,176 subjects in the cohort with low birth weight $(<2,500$ grams), and 151 had a birth weight of less than 1,500 grams. In all, 2,931 subjects were born preterm (< 37 weeks), and 986 had a gestational duration of 32 weeks or less. We considered cohort subjects as cases if they had a main discharge diagnosis of asthma or COPD.

Results: For any obstructive airways disease, there was a statistically significant increase in risk with decreasing birth weight when males and females were analyzed together.
Compared to women born at term, women born before 33 weeks of gestation had an almost threefold increase in risk for any obstructive airways disease. For fetal growth, the association was u-shaped rather than linear.

\section{9}

\section{LOW MATERNAL LEVELS OF FIRST- TRIMESTER PAPP-A AND FREE B-HCG ARE ASSOCIATED WITH AN INCREASED RISK OF ADVERSE NEONATAL OUTCOME}

\author{
I. Kirkegaard ${ }^{1,2}$, N. Uldbjerg ${ }^{2}$, T.B. Henriksen ${ }^{1,3}$ \\ ${ }^{1}$ Perinatal Epidemiolgoy Research Unit, ${ }^{2}$ Dept. \\ Obstetrics and Gynecology, ${ }^{3}$ Dept. Pediatrics, \\ Aarhus University Hospital, Skejby, \\ Aarhus, Denmark
}

Background and aim: Pregnancy-associated plasma protein-A (PAPP-A) and free beta-human chorionic gonadotropin ( $\beta-\mathrm{hCG}$ ) are highly efficient in first-trimester screening for Down syndrome. In addition, studies have found them associated to preterm delivery, pre-eclampsia and low birth weight, but the association to neonatal outcomes is only sparsely elucidated. The aim of the current study was evaluate PAPP-A and free $\beta-h C G$ in relation to adverse neonatal outcomes.

Methods: 9450 singleton pregnant women at Aarhus University Hospital were included between January 2005 and December 2007. PAPP-A and free $\beta$-hCG were measured in the first trimester. Neonatal outcomes were obtained from specific registration forms filled out by the doctors responsible for admission and discharge from the neonatal intensive care unit (NICU). The outcomes explored in this study were admission to NICU, neonatal jaundice and hypoglycemia.

Results: PAPP-A below 0.4 MoM was significantly associated with admission to NICU (OR 1.6; $95 \% \mathrm{Cl}$, 1.2 - 2.0), and the same applied to free $\beta$-hCG below $0.4 \mathrm{MoM}$ (OR 1.5; 95\% Cl, $1.1-2.1$ ). The results could not be explained by preterm delivery or low birth weight as the results remained unchanged when restricting to deliveries in gestational weeks 39 and 40 and when adjusting for birth weight. Furthermore, we found significant associations between PAPP-A and both hypoglycemia and jaundice and between free $\beta-h C G$ and jaundice.

Conclusion: Both low PAPP-A and free $\beta$-hCG were associated with an increased risk of admission to NICU. The results could not be explained by either preterm delivery or low birth weight. 